\title{
COMPORTAMENTO ESTOMÁTICO E POTENCIAL DA ÁGUA DA FOLHA EM TRÊS ESPÉCIES LENHOSAS CULTIVADAS SOB ESTRESSE HÍDRICO ${ }^{1}$
}

\author{
Elizamar Ciríaco da Silva ${ }^{2,3}$ \\ Rejane Jurema Mansur Custódio Nogueira² \\ André Dias de Azevedo Neto ${ }^{4}$ \\ Venezio Felipe dos Santos ${ }^{5}$
}

Recebido em 20/01/2002. Aceito em 17/07/2002

RESUMO - (Comportamento estomático e potencial da água da folha em três espécies lenhosas cultivadas sob estresse hídrico). Com o objetivo de avaliar o comportamento estomático e o potencial da água da folha em plantas jovens de Mimosa caesalpiniifolia, Enterolobium contortisiliquum e Tabebuia aurea submetidas a estresse hídrico, desenvolveu-se um experimento em casa de vegetação no Laboratório de Fisiologia Vegetal da UFRPE. As plântulas foram submetidas a dois tratamentos hídricos (100\% da CP e 50\% da CP) e aos 51, 7, 13 e 22 dias de estresse hídrico, foram avaliadas a transpiração (E), a resistência difusiva (Rs), a temperatura da folha (Tfol), a temperatura do ar (Tar), umidade relativa do ar (UR), a radiação fotossinteticamente ativa (PAR) e o déficit de pressão de vapor (DPV) às $7 \mathrm{~h}, 9 \mathrm{~h}, 12 \mathrm{~h}$ e $15 \mathrm{~h}$. Após 30 dias de estresse, foi medido o potencial da água da folha ( $\Psi \mathrm{w})$ entre 9-10 horas. Os maiores valores de $\mathrm{E}$ foram registrados entre $9-12 \mathrm{~h}$ para todas as espécies, sendo mais baixos nas plantas estressadas. O oposto foi verificado para Rs, com diferenças na magnitude dos valores entre espécies e horários de avaliação. A Rs das plantas estressadas de E. contortisiliquum foi influenciada pela Tar e de $M$. caesalpiniifolia pela UR e PAR. O $\Psi$ w decresceu com o estresse, com reduções de até $155 \%$. A Tfol não se mostrou bom indicador dos efeitos do estresse hídrico sobre as espécies.

Palavras-chave - potencial hídrico, resistência difusiva, transpiração

\begin{abstract}
Estomatal behavior and leaf water potential in three wood species cultivated under water stress). The aim of this work was to evaluate the estomatal behavior and the leaf water potential in Mimosa caesalpiniifolia, Enterolobium contortisiliquum and Tabebuia aurea young plants cultivated under water stress. This work was performed in greenhouse of the Laboratório de Fisiologia Vegetal of the Universidade Federal Rural de Pernambuco, Brazil. Seedlings were submitted to two water treatment (100\% and $50 \%$ pot capacity). On the $5^{\text {th }}, 7^{\text {th }}, 13^{\text {th }}$ and $22^{\text {th }}$ days of water treatments, the transpiration (E), diffusive resistance (Rs), leaf temperature (Tfol), air temperature (Tar), relative air humidity (UR), photossynthetically active radiation (PAR) and vapour pressure deficit (DPV) were evaluated at $7,9,12,15 \mathrm{~h}$. After $30^{\text {th }}$ days the leaf water potential $(\Psi \mathrm{w})$ was measured, between $9-10 \mathrm{~h}$. The
\end{abstract}

1 Parte da Dissertação de Mestrado da primeira Autora; auxílio CNPq

Programa de Pós-Graduação em Botânica

Rua Salvador Mendonça, 175, Jardim Brasil, CEP 53290-060, Olinda, PE, Brasil

Departamento de Química (UFRPE) - Recife, PE, Brasil

Pesquisador do IPA 


\begin{abstract}
highest values of E were registered between 9-12h for all the species, although the stressed plants presented inferior values compared to the control plants. The opposite was verified for the Rs, however the differences in the magnitude and values among species and hours of evaluated were observed. The stomatal behavior of the stressed plants of E. contortisiliquum was influenced by the Tar and the M. caesalpiniifolia was influenced by the UR and PAR. The $\Psi_{\mathrm{w}}$ decreased with the placing of stress, with reduction as much as $155 \%$. The leaf temperature did not show a good indicator on the effects of water stress on the species studied.
\end{abstract}

Key words - water potential, diffusive resistance, transpiration

\section{Introdução}

No Nordeste brasileiro, nas áreas de caatinga, é comum a deficiência hídrica no solo devido ao regime pluviométrico anual irregular e escasso $(250-500 \mathrm{~mm})$, associado com altas temperaturas entre $26-27^{\circ} \mathrm{C}$ (Mendes 1986) e forte evaporação causada pela demanda evaporativa do ar e de altos níveis de radiação (Larcher 2000). Essa condição delimita duas estações bem distintas: uma estação chuvosa curta, que dura de 3 a 5 meses, e uma longa estação seca, que dura de 7 a 9 meses, podendo se estender por anos (Mendes 1986; AndradeLima 1989).

As plantas ocorrentes nessas áreas respondem ao estresse ambiental de formas diferenciadas e essas respostas têm sido foco do estudo de ecologistas e fisiologistas (Hopkins 1995; Ferri 1979; Sampaio et al. 1998; Nogueira et al. 1998a; Nogueira et al. 1998c; Mansur et al. 2000). Dentre as espécies que podem ser encontradas nessas áreas, destacam-se a Mimosa caesalpiniifolia Benth (sabiá) e o Enterolobium contortisiliquum (Vell.) Morong. (tamboril), bem como a Tabebuia aurea (Manso) Benth e Hook. (craibeira). As duas primeiras pertencem à família Fabaceae, subfamília Mimosoideae e a craibeira pertence à família Bignoniaceae. Mimosa caesalpiniifolia é espécie comum nas áreas de caatinga do Ceará e Piauí (Rizzini, 1986), Tabeuia aurea em Pernambuco, Paraíba, Rio Grande do Norte e Ceará e Enterolobium contortisiliquum, embora tenha se estabelecido nas florestas pluviais, também pode ser encontrado desde o litoral até o Sertão do Ceará (Tigre 1964).
Possuem grande potencial madeireiro, são utilizadas na ornamentação de ruas, empregadas para fins de reflorestamento para regeneração de áreas degradadas e além disso, $M$. caesalpiniifolia e E. contortisiliquum são espécies forrageiras (Rizzini 1986; Tigre 1964; Braga 1976; Gomes 1977; Mendes 1989).

A água é um dos fatores ambientais determinantes da diversidade produtiva dos vegetais, podendo comprometer as funções vitais ou estimular reações adaptativas que capacitem as plantas a sobreviverem por períodos longos de estresse hídrico (Diniz 1999). O déficit de água nos tecidos, causado pela excessiva demanda evaporativa ou pelo suprimento de água no solo limitado afetam todos os aspectos do crescimento e desenvolvimento dos vegetais (Krieg, 1993).

Devido à limitada reserva de água nas folhas e a taxa potencial de transpiração, a regulação da abertura estomática para restringir os danos nos tecidos, como resultado da desidratação, é de grande importância para a planta (Ferreira 1997). O fechamento dos estômatos como tentativa de manter o conteúdo hídrico favorável nos tecidos por maior tempo possível, é uma das primeiras linhas de defesa contra a dessecação. Essa redução da abertura do poro estomático, porém, restringe a troca de gases entre o interior da folha e a atmosfera causando diminuição na assimilação de $\mathrm{CO}_{2}$ que é utilizado no processo fotossintético (Krieg 1993; Larcher 2000).

A transpiração das plantas tende a aumentar quando o gradiente de pressão de vapor entre a folha e o ar atmosférico se eleva. Esse gradiente é calculado em função da temperatura e da umidade relativa do ar, que também pode 
aumentar quando ocorre o secamento do solo (Hopkins 1995). Alguns autores verificaram que o aumento do déficit de pressão de vapor, quando a planta se encontra sob estresse hídrico, reduz o fluxo de vapor d'água do interior da câmara sub-estomática para a atmosfera, causando depressão na transpiração (Gucci et al. 1996; Thomas et al. 2000). No entanto, Nogueira et al. (2001) observaram que a depressão na taxa de transpiração ocasionada pelo fechamento parcial ou total dos estômatos, em aceroleiras submetidas a estresse hídrico, foi mais influenciada pelo potencial da água da folha do que por outros fatores ambientais, como umidade relativa ou temperatura do ar.

O potencial de água da folha também diminui com o declínio da disponibilidade de água no solo, levando à perda da turgescência e ao fechamento estomático (Mansur e Barbosa 2000). O estado energético da água na planta resulta da interação da demanda evaporativa atmosférica com o potencial de água no solo, densidade e distribuição do sistema radicular e processos fisiológicos (Kramer 1969). Os valores de potencial hídrico foliar nas espécies ocorrentes em regiões semi-áridas geralmente são bem menores do que os encontrados em espécies de florestas pluviais, entretanto esses valores são diferenciados de acordo com as características de cada espécie. Nogueira et al. (1998a) encontraram variações nos valores do potencial hídrico foliar em três espécies ocorrentes em áreas de caatinga, sugerindo que as diferenças quantitativas são influenciadas pelas adaptações morfológicas peculiares a cada espécie.

Embora essas informações sejam oferecidas na literatura de forma bem generalizada, não são encontrados registros de estudos envolvendo as trocas gasosas e relações hídricas com o sabiá, craibeira e o tamboril. Fazem-se necessárias pesquisas, não apenas com estas, mas com outras espécies que ofereçam valor econômico, face à degradação e à pouca importância dada ao ecossistema caatinga. Diante do exposto, o objetivo do presente trabalho foi avaliar o comportamento estomático e o potencial de água na folha em plantas jovens de sabiá, craibeira e tamboril quando submetidas a estresse hídrico, correlacionando-os aos fatores ambientais.

\section{Material e métodos}

O experimento foi conduzido em casa de vegetação e Laboratório de Fisiologia Vegetal, Departamento de Biologia, Área de Botânica, da Universidade Federal Rural de Pernambuco, Brasil, no período de março a julho/2000.

Para a condução deste experimento, foram utilizadas mudas com aproximadamente dois meses de idade de Mimosa caesalpiniifolia Benth., Enterolobium contortisiliquum (Vell.) Morong. e Tabebuia aurea (Manso) Benth. \& Hook. A propagação foi feita através de sementes coletadas de área de caatinga em Xingó, BA (E. contortisiliquum e T. aurea) e cedidas pelo Departamento de Ciências Florestais da UFRPE (M. caesalpiniifolia) procedentes da Estação de Fomento do IBAMA, Município de Nísia Floresta, RN.

As sementes foram colocadas para germinar em bandejas com capacidade para 3L, contendo como substrato areia lavada não esterilizada e colocadas em câmara úmida, visando minimizar a perda de água por evaporação.

Após a emergência, com aproximadamente $8 \mathrm{~cm}$ alt., foram selecionadas 10 plântulas de cada espécie e as mesmas foram transferidas para sacos de polietileno contendo $3 \mathrm{~kg}$ de solo classificado como Neossolo Regolítico, coletado na profundidade de $0-40 \mathrm{~cm}$, procedente de uma região de baixio da Estação Experimental do IPA, no município de Caruaru, PE. Para simular as condições da caatinga, optou-se por não adubar o solo. A superfície dos vasos foi revestida com plástico, para evitar a perda de água do solo por evaporação.

Foi utilizado um delineamento experimental em blocos ao acaso, em esquema fatorial de 
$3 \times 2$, correspondendo a três espécies, dois tratamentos hídricos (controle - 100\% da capacidade de pote; estressado - 50\% da capacidade de pote), e quatro repetições. A capacidade de pote (CP) foi adotada como o conteúdo de água retida pelo solo após sofrer saturação e conseqüente ação da gravidade, até o cessamento da drenagem, segundo Souza et al. (2000).

Durante o período de aclimatação (aproximadamente um mês), todos os vasos foram irrigados diariamente, mantendo-se na capacidade de pote. Após este período, um grupo de plantas continuou recebendo esta quantidade de água (controle), e o outro grupo passou a receber $50 \%$ da capacidade de pote (estressado), sendo a reposição da água feita em função da água perdida por evapotranspiração de um dia para o outro, a qual foi verificada através da diferença no peso dos vasos, utilizando-se balança marca Filizola, com capacidade para $10 \mathrm{~kg}$.

Aos 5, 7, 13 e 22 dias após a diferenciação dos tratamentos hídricos, foram mensuradas a transpiração (E) e a resistência difusiva (Rs) em folhas maduras e completamente expandidas, localizadas no terço médio das plantas, utilizando-se um porômetro de equilíbrio dinâmico da LICOR (modelo L!-1600). Simultaneamente, foram registrados os valores de umidade relativa do ar (UR), temperatura foliar (Tfol), temperatura do ar (Tar) e a radiação fotossinteticamente ativa (PAR) sendo estes últimos através de termopares e sensores quânticos existentes no mesmo equipamento. As medidas foram feitas às $7 \mathrm{~h}, 9 \mathrm{~h}, 12 \mathrm{~h}$ e $15 \mathrm{~h}$. Com os dados de Tar e UR, foi calculado o déficit de pressão de vapor (DPV), para os mesmos horários, segundo Vianello \& Alves (1988).

Após 30 dias, foi avaliado o potencial da água da folha utilizando-se as mesmas folhas usadas para as medidas porométricas, através do uso da câmara de pressão de Scholander (Scholander 1964). As medidas foram tomadas entre as 9 e 10 horas. Para evitar a perda de água por transpiração, as folhas foram cortadas na inserção do pecíolo, envoltas em filme plástico e acondicionadas sob refrigeração. Imediatamente após, foram levadas ao Laboratório de Fisiologia Vegetal para o procedimento das medidas.

Os dados foram submetidos à análise de variância e correlação simples. As médias foram comparadas pelo teste de Tukey a uma probabilidade de $5 \%$.

\section{Resultados e discussão}

Durante o período experimental a temperatura do ar variou em média de $27,32^{\circ} \mathrm{C}$ a $30,99^{\circ} \mathrm{C}$, sendo maior nos horários de maior radiação solar. A umidade relativa do ar (UR) variou em média de $51,63 \%$ a $76 \%$ e a radiação fotossinteticamente ativa (PAR), de $233,92 \mu \mathrm{mol} . \mathrm{m}^{-2} . \mathrm{s}^{-1}$ a $825,96 \mu \mathrm{mol} . \mathrm{m}^{-2} . \mathrm{s}^{-1}$, tendo esse valor mais alto sido registrado às 15 horas no sétimo dia de tratamento hídrico. O déficit de pressão de vapor entre a folha e o ar variou de $0,948 \mathrm{kPa}$ a $1,975 \mathrm{kPa}$. O maior valor de DPV registrado culmina com o menor valor de UR e maior PAR (Fig.1).

De forma geral, as plantas transpiraram mais nos horários entre 9 e 12 horas, quando a temperatura do ar e a radiação solar apresentavam-se mais elevadas e baixa umidade relativa (Fig. 1).

Nas plantas de $M$. caesalpiniifolia, os valores de E variaram de $1,55 \mu \mathrm{mol} . \mathrm{m}^{-2} \cdot \mathrm{s}^{-1}$ a $10,44 \mu \mathrm{mol} \cdot \mathrm{m}^{-2} \cdot \mathrm{s}^{-1}$ para as controle e de $1,03 \mu \mathrm{mol} . \mathrm{m}^{-2} \cdot \mathrm{s}^{-1}$ a $2,99 \mu \mathrm{mol} \cdot \mathrm{m}^{-2} . \mathrm{s}^{-1}$ nas submetidas a deficiência hídrica (Fig. 2). Essa espécie reduziu a transpiração aos 5 dias de tratamento hídrico, apresentando valores mais baixos de $\mathrm{E}$ em todos os horários nas plantas estressadas. Essa redução no horário de maior demanda evaporativa foi em média de 70,12\%. Aos sete dias essa redução chegou a 77,78\% às 9 horas. Aos 13 e 22 dias foi de $70,21 \%$ e $52,12 \%$ às 12 horas, respectivamente (Fig. 2A, D, G e J).

Não houve diferença significativa entre os 


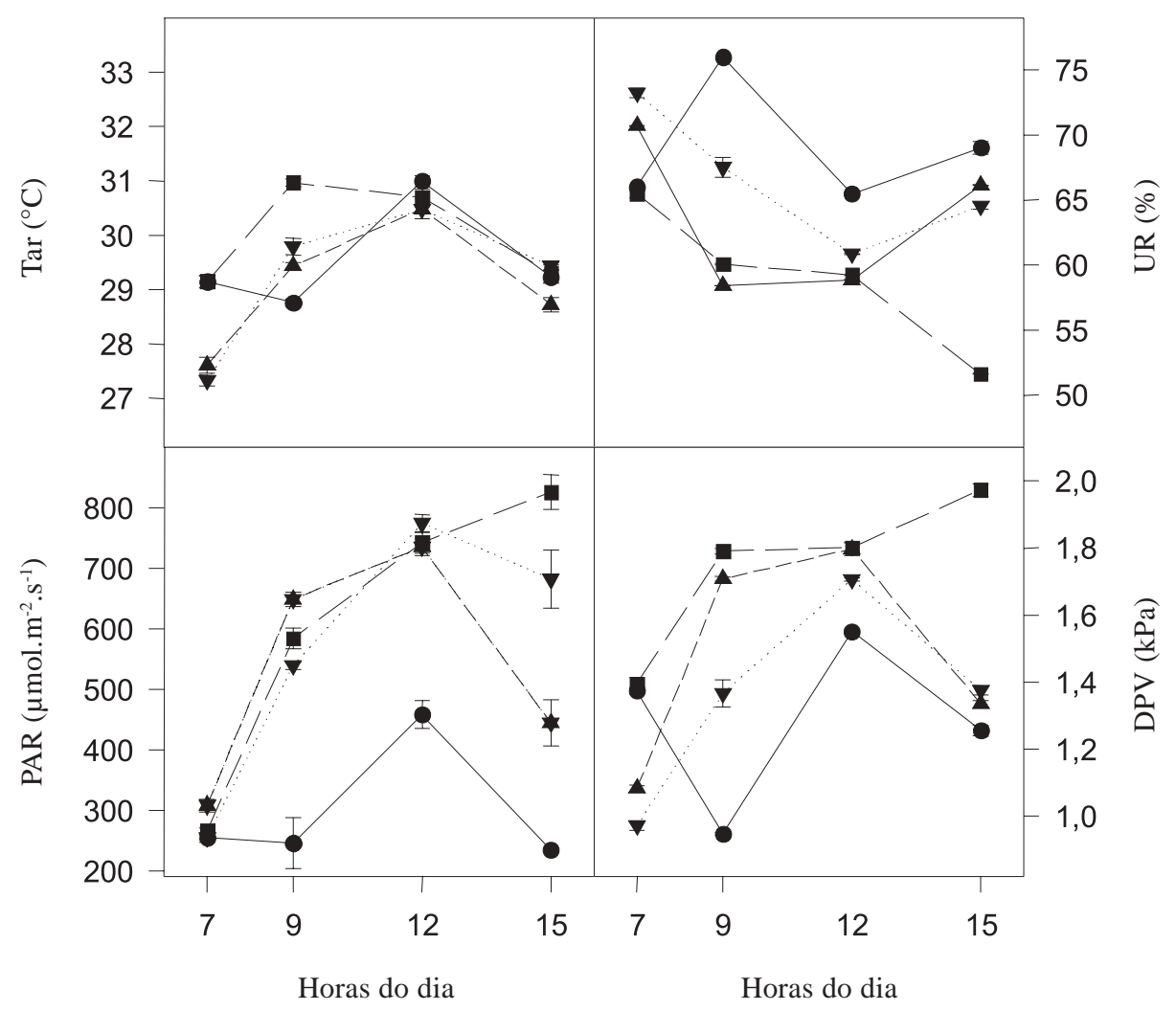

Figura 1. Curso diário da temperatura do ar (Tar), umidade relativa do ar (UR), radiação fotossinteticamente ativa (PAR) e déficit de pressão de vapor (DPV) por ocasião das medidas porométricas em plantas jovens de sabiá, tamboril e craibeira cultivadas em casa de vegetação aos $5,7,13$ e 22 dias após a diferenciação dos tratamentos hídricos. —

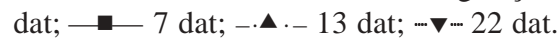

tratamentos hídricos aos 22 dias, às 15 horas, provavelmente em virtude da redução da velocidade de absorção de água pelo sistema radicular não suprir a demanda atmosférica nas plantas controle (Fig. 2J).

Quanto ao E. contortisiliquum, este também se mostrou sensível ao estresse hídrico no que se refere à redução da transpiração. As plantas estressadas reduziram as taxas de E em quase todos os horários, sendo exceção apenas às 15 horas aos 5 e 13 dias de tratamento (Fig. 2B, E, $\mathrm{H}$ e L).O valor mais elevado de E para essa espécie nas plantas controle foi de $4,22 \mu \mathrm{mol} . \mathrm{m}^{-2}$ s $^{-1}$ às 9 horas aos 7 dias, e o mais baixo foi verificado às 15 horas aos 5 dias $\left(0,87 \mu \mathrm{mol} \cdot \mathrm{m}^{-2} \cdot \mathrm{s}^{-1}\right)$. Nas plantas estressadas o maior valor foi de $2,57 \mu \mathrm{mol} . \mathrm{m}^{-2} . \mathrm{s}^{-1}$ às 9 horas e o mais baixo de $0,50 \mu \mathrm{mol} \cdot \mathrm{m}^{-2} \cdot \mathrm{s}^{-1}$ às 15 horas aos 5 dias de tratamento. A redução na transpiração das plantas estressadas foi de até $42,42 \%, 65,40 \%$ e $58,25 \%$ aos 7,13 e 22 dias de estresse hídrico.

Independente dos tratamentos hídricos, aos 5 e 22 dias, as plantas de T. aurea apresentaram valores de transpiração mais elevados às 12 horas, enquanto que aos 7 e 13 dias os maiores valores foram observados às 9 horas (Fig. 2C, $\mathrm{F}$, I e M). Esses variaram de 2,62 $\mu \mathrm{mol} \cdot \mathrm{m}^{-2} \cdot \mathrm{s}^{-1} \mathrm{a}$ $10,18 \mu \mathrm{mol} \cdot \mathrm{m}^{-2} \cdot \mathrm{s}^{-1}$ nas plantas controle e de $1,20 \mu \mathrm{mol} . \mathrm{m}^{-2} . \mathrm{s}^{-1}$ a $8,75 \mu \mathrm{mol} . \mathrm{m}^{-2} . \mathrm{s}^{-1}$ nas estressadas. Aos cinco dias de diferenciação dos tratamentos hídricos, as plantas estressadas de T. aurea, quando comparadas com as plantas controle, só restringiram a transpiração às 12 


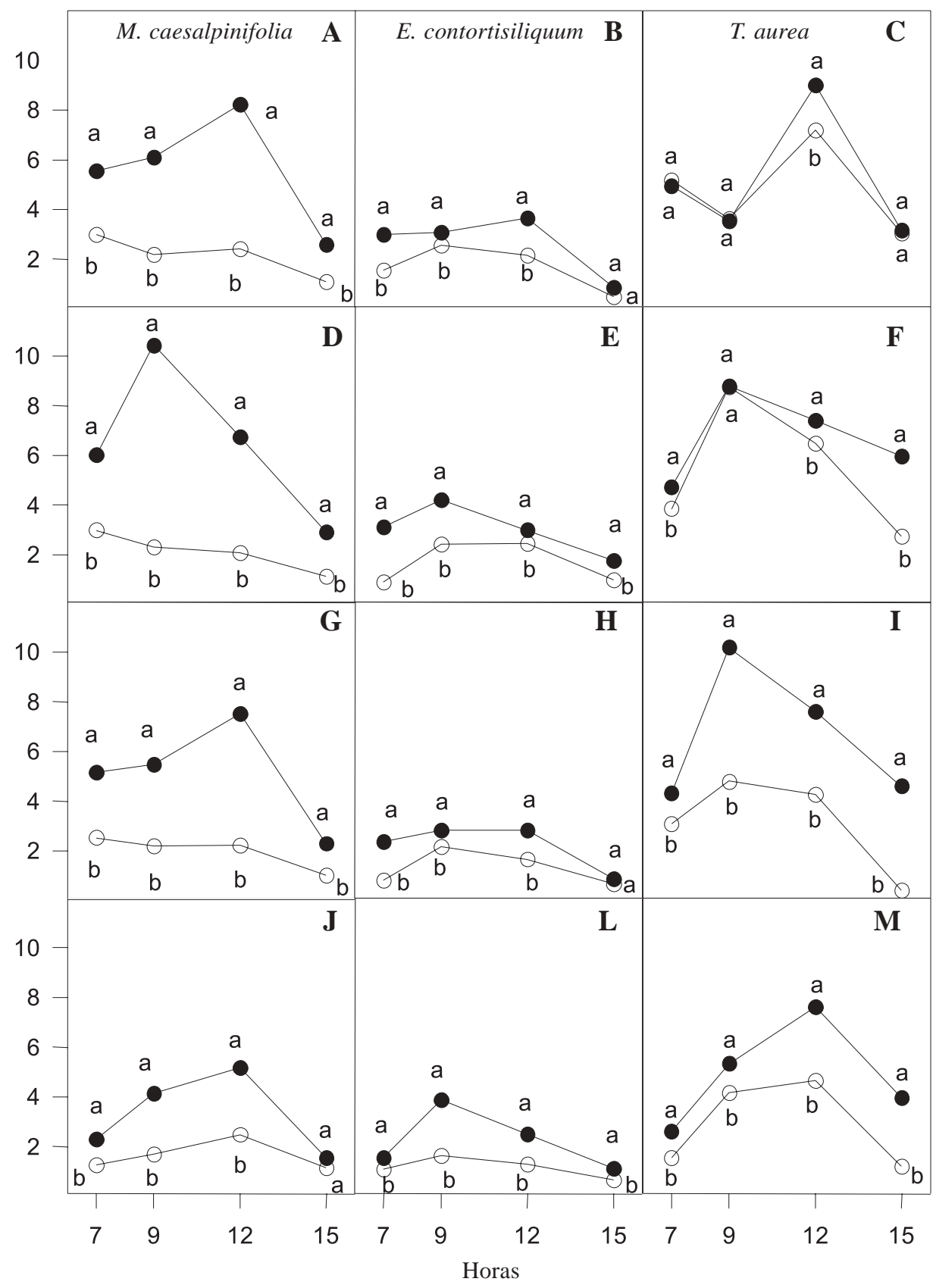

Figura 2. Curso diário da transpiração em plantas jovens de Mimosa caesalpiniifolia, Enterolobium contortisiliquum e Tabebuia aurea após 5 (A, B, C), 7 (D, E, F), 13 (G, H, I) e 22 (J, L, M) dias de tratamentos hídricos em casa de vegetação. Letras iguais não diferem entre os tratamentos pelo teste de Tukey ao nível de probabilidade de 5\%.—— Controle; —_- Estresse. 
horas (Fig. 2C).

Aos 7 dias, as plantas estressadas apresentaram valores menores de $\mathrm{E}$ do que as controle em quase todos os horários. Apenas às 9 horas não houve diferença significativa entre os valores de E para plantas controle e estressadas (Fig. 2F), no entanto, aos 13 e 22 dias houve diminuição significativa da taxa de transpiração em todas as avaliações (Fig. 2I e $2 \mathrm{M}$ ), com reduções de até $52,89 \%$ e $39,06 \%$ respectivamente.

Esses resultados assemelham-se aos encontrados por Nogueira et al.(1998a), onde os autores observaram reduções de $69,66 \%$, $82,41 \%$ e $69,66 \%$ na transpiração de plantas estressadas em relação às controle, em três espécies da caatinga, Senna martiana, Parkinsonia aculeata e Senna occidentalis, respectivamente e também aos encontrados por Nogueira et al.(2001), onde os autores verificaram que períodos crescentes de estresse hídrico causaram decréscimos na taxa transpiratória de aceroleiras cultivadas em casa de vegetação após suspensão de rega. Prado et al. (1994) verificaram redução na taxa de transpiração em plantas de 6 meses de idade de Copaifera langsdorfii, espécie do cerrado, quando estas foram submetidas a estresse hídrico por suspensão de rega. Resultados semelhantes foram encontrados para Stryphnodendron adstringens com seis meses de idade, espécie também do cerrado por Rocha \& Moraes (1997). Os autores verificaram decréscimo na taxa de transpiração quando estas foram submetidas a estresse hídrico por suspensão de rega, atingindo no $30^{\circ}$ dia o valor aproximado de $0,1 \mu \mathrm{mol} \cdot \mathrm{m}^{-2} \cdot \mathrm{s}^{-1}$ (fechamento estomático). Plantas jovens de Caesalpinia pyramidalis, Caesalpinia ferrea, Senna martiana e Senna spectabilis apresentaram valores de $\mathrm{E}$ de 0,66 ; 0,$65 ; 0,54$ e $0,66 \mu \mathrm{mol} \mathrm{m}^{-2} . \mathrm{s}^{-1}$, respectivamente, após 14 dias de suspensão de rega (Mansur \& Barbosa 2000). Comparando os valor encontrados por Mansur \& Barbosa.(2000) com os encontrados no presente trabalho, observa-se que o nível de estresse atribuído às plantas não impediu que as mesmas transpirassem, mesmo nos horários de maior demanda evaporativa, permitindo dessa forma a absorção de $\mathrm{CO}_{2}$ para a fotossíntese.

A redução na transpiração nas plantas estressadas de $M$. caesalpiniifolia $e$ E. contortisiliquum não se correlacionou com a temperatura do ar (Tar), com a umidade relativa do ar (UR), com a radiação fotossinteticamente ativa (PAR), nem com o déficit de pressão de vapor (DPV); apenas as plantas estressadas de $T$. aurea apresentaram correlação positiva com a temperatura do ar (Tab. 1, 2 e 3). Isso demonstra que a disponibilidade de água no solo para essas espécies foi o fator que realmente influenciou a redução da transpiração. Verifica-se ainda correlação inversa entre a transpiração e a resistência dos estômatos nas plantas de M. caesalpiniifolia

Tabela 1. Matriz de correlação simples entre a resistência difusiva (Rs), a transpiração (E), o potencial hídrico foliar $\left(\Psi_{\mathrm{w}}\right)$, a temperatura foliar (Tfol), a temperatura do ar (Tar), a umidade relativa do ar (UR) e a radiação fotossinteticamente ativa (PAR) em plantas jovens de Mimosa caesalpiniifolia, submetidas a dois regimes hídricos em casa de vegetação.

\begin{tabular}{lcc}
\hline Variáveis & Controle & Estresse \\
\hline Tfol X Tar & $0,9992^{* *}$ & $0,9984^{* *}$ \\
TfolX UR & $-0,4922^{\mathrm{NS}}$ & $-0,6499^{* *}$ \\
Tfol X PAR & $0,4244^{\mathrm{NS}}$ & $0,5369^{*}$ \\
Tfol X DPV & $0,7261^{* *}$ & $0,8143^{* *}$ \\
Tfol XRs & $-0,4194^{\mathrm{NS}}$ & $0,1224^{\mathrm{NS}}$ \\
Tfol X E & $0,6932^{* *}$ & $0,2661^{\mathrm{NS}}$ \\
E X Tar & $0,6985^{* *}$ & $0,2942^{\mathrm{NS}}$ \\
E X UR & $-0,2269^{\mathrm{NS}}$ & $-0,0010^{\mathrm{NS}}$ \\
E X PAR & $0,0662^{\mathrm{NS}}$ & $-0,2472^{\mathrm{NS}}$ \\
E X DPV & $0,4265^{\mathrm{NS}}$ & $0,0913^{\mathrm{NS}}$ \\
E X Rs & $-0,8224^{* *}$ & $-0,7869^{* *}$ \\
Rs X Tar & $-0,4230^{\mathrm{NS}}$ & $0,0832^{\mathrm{NS}}$ \\
Rs X UR & $-0,1848^{\mathrm{NS}}$ & $-0,5578^{*}$ \\
Rs X PAR & $0,3420^{\mathrm{NS}}$ & $0,5874^{*}$ \\
Rs X DPV & $-0,0062^{\mathrm{NS}}$ & $0,4552^{\mathrm{NS}}$ \\
\hline
\end{tabular}

* Significativo pelo teste $\mathrm{F}(\mathrm{P}<0,05$; ** Altamente significativo pelo teste $F(P<0,05)$; Ns Não significativo pelo teste $F$ $(\mathrm{P}<0,05)$ 
Tabela 2. Matriz de correlação simples entre a resistência difusiva (Rs), a transpiração (E), o potencial hídrico foliar $(\Psi \mathrm{w})$, a temperatura foliar (Tfol), a temperatura do ar (Tar), a umidade relativa do ar (UR) e a radiação fotossinteticamente ativa (PAR) em plantas jovens de Enterolobium contortisiliquum, submetidas a dois regimes hídricos em casa de vegetação.

\begin{tabular}{lcc}
\hline Variáveis & Controle & Estresse \\
\hline Tfol X Tar & $0,9974^{* *}$ & $09945^{* *}$ \\
TfolX UR & $-0,6633^{* *}$ & $-0,5438^{*}$ \\
Tfol X PAR & $0,6946^{* *}$ & $0,6422^{* *}$ \\
Tfol X DPV & $0,8049^{* *}$ & $0,7515^{* *}$ \\
Tfol XRs & $-0,2939^{\mathrm{Ns}}$ & $-0,5239^{*}$ \\
Tfol X E & $0,6530^{* *}$ & $-0,2215^{\mathrm{NS}}$ \\
E X Tar & $0,6684^{* *}$ & $-0,2296^{\mathrm{NS}}$ \\
E X UR & $-0,1637^{\mathrm{NS}}$ & $0,0113^{\mathrm{NS}}$ \\
E X PAR & $0,2250^{\mathrm{NS}}$ & $-0,1174^{\mathrm{NS}}$ \\
E X DPV & $0,3117^{\mathrm{NS}}$ & $-0,0870^{\mathrm{NS}}$ \\
E X Rs & $-0,8542^{* *}$ & $0,2085^{\mathrm{NS}}$ \\
Rs X Tar & $-0,3022^{\mathrm{NS}}$ & $-0,4985^{*}$ \\
Rs X UR & $-0,1950^{\mathrm{NS}}$ & $-0,1259^{\mathrm{NS}}$ \\
Rs X PAR & $0,0239^{\mathrm{NS}}$ & $-0,2660^{\mathrm{NS}}$ \\
Rs X DPV & $0,0701^{\mathrm{NS}}$ & $-0,0722^{\mathrm{NS}}$ \\
\hline
\end{tabular}

* Significativo pelo teste $\mathrm{F}(\mathrm{P}<0,05)$; ** Altamente significativo pelo teste $\mathrm{F}(\mathrm{P}<0,05)$; ${ }^{N}$ Não significativo pelo teste $\mathrm{F}(\mathrm{P}<0,05)$

e T. aurea, tanto para as que estavam sob boa disponibilidade hídrica no solo, como para as estressadas. No caso do E. contortisiliquum essa correlação só foi evidente para as plantas controle.

Com relação à resistência dos estômatos à difusão do vapor de água (Rs), de forma geral observou-se que os maiores valores foram registrados às 15 horas para todas as espécies. Analisando individualmente, verificou-se que as plantas de $M$. caesalpiniifolia apresentaram diferença significativa para a Rs entre os tratamentos hídricos aplicados em todos os horários e épocas de avaliação (Fig. 3). O estresse hídrico aumentou a Rs nessa espécie em até 304,10\% em relação às plantas do tratamento controle. O maior valor de Rs nas plantas estressadas foi de 777,25 s.m-1 quando submetidas a 7 dias de estresse, e o menor foi de 160,0 s.m ${ }^{-1}$, aos 5 dias. Para as plantas do
Tabela 3. Matriz de correlação simples entre a resistência difusiva (Rs), a transpiração (E), o potencial hídrico foliar $\left(\Psi_{\mathrm{w}}\right)$, a temperatura foliar (Tfol), a temperatura do ar (Tar), a umidade relativa do ar (UR) e a radiação fotossinteticamente ativa (PAR) em plantas jovens de Tabebuia aurea, submetidas a dois regimes hídricos em casa de vegetação.

\begin{tabular}{lcc}
\hline Variáveis & Controle & Estresse \\
\hline Tfol X Tar & $0,9505^{* *}$ & $0,9979 * *$ \\
TfolX UR & $-0,3492^{\mathrm{NS}}$ & $-0,5169^{*}$ \\
Tfol X PAR & $0,5629^{*}$ & $0,5174^{*}$ \\
Tfol X DPV & $0,5723^{*}$ & $0,7339^{*}$ \\
Tfol XRs & $-0,3976^{\mathrm{NS}}$ & $-0,2338^{\mathrm{NS}}$ \\
Tfol X E & $0,6008^{*}$ & $0,7104^{*}$ \\
E X Tar & $0,7052^{*}$ & $0,7118^{* *}$ \\
E X UR & $-0,6998^{*}$ & $-0,2834^{\mathrm{NS}}$ \\
E X PAR & $0,4945^{\mathrm{NS}}$ & $0,2212^{\mathrm{NS}}$ \\
E X DPV & $0,8045^{* *}$ & $0,4746^{\mathrm{NS}}$ \\
E X Rs & $-0,8142^{* *}$ & $-0,5882^{*}$ \\
Rs X Tar & $-0,5215^{*}$ & $-0,2652^{\mathrm{NS}}$ \\
Rs X UR & $0,3265^{\mathrm{NS}}$ & $-0,2041^{\mathrm{NS}}$ \\
Rs X PAR & $-0,0669^{\mathrm{NS}}$ & $0,3288^{\mathrm{NS}}$ \\
Rs X DPV & $-0,4373^{\mathrm{NS}}$ & $0,0678^{\mathrm{NS}}$ \\
\hline
\end{tabular}

* Significativo pelo teste $\mathrm{F}(\mathrm{P}<0,05) ; * *$ Altamente significativo pelo teste $\mathrm{F}(\mathrm{P}<0,05)$; ${ }^{N}$ Não significativo pelo teste $\mathrm{F}(\mathrm{P}<0,05)$

tratamento controle, o maior valor médio foi de $322,00 \mathrm{~s} . \mathrm{m}^{-1}$, aos 22 dias de tratamento e o menor foi de 49,25 s.m-1, aos 5 dias de avaliação. Observou-se correlação positiva com a radiação fotossinteticamente ativa (PAR) e negativa com a UR apenas para as plantas cultivadas sob estresse (Tab. 1).

E. contortisliquum apresentou comportamento similar ao de $M$. caesalpiniifolia quando em condições de estresse hídrico. Só não houve diferença significativa para a Rs entre plantas controle e estressada às 9 horas no quinto dia após diferenciação dos tratamentos. Os maiores valores de Rs foram registrados sempre às 15 horas, tanto para as plantas controle como para as plantas estressadas (Fig. 2B, E, H, L).

$\mathrm{O}$ estresse hídrico aumentou a Rs em até $107,49 \% ; 274,19 \% ; 224,25 \%$ e $120,39 \%$ aos 5 , 7,13 e 22 dias, respectivamente. Para as plantas controle o maior valor de Rs foi de 609,0 s.m-1, 


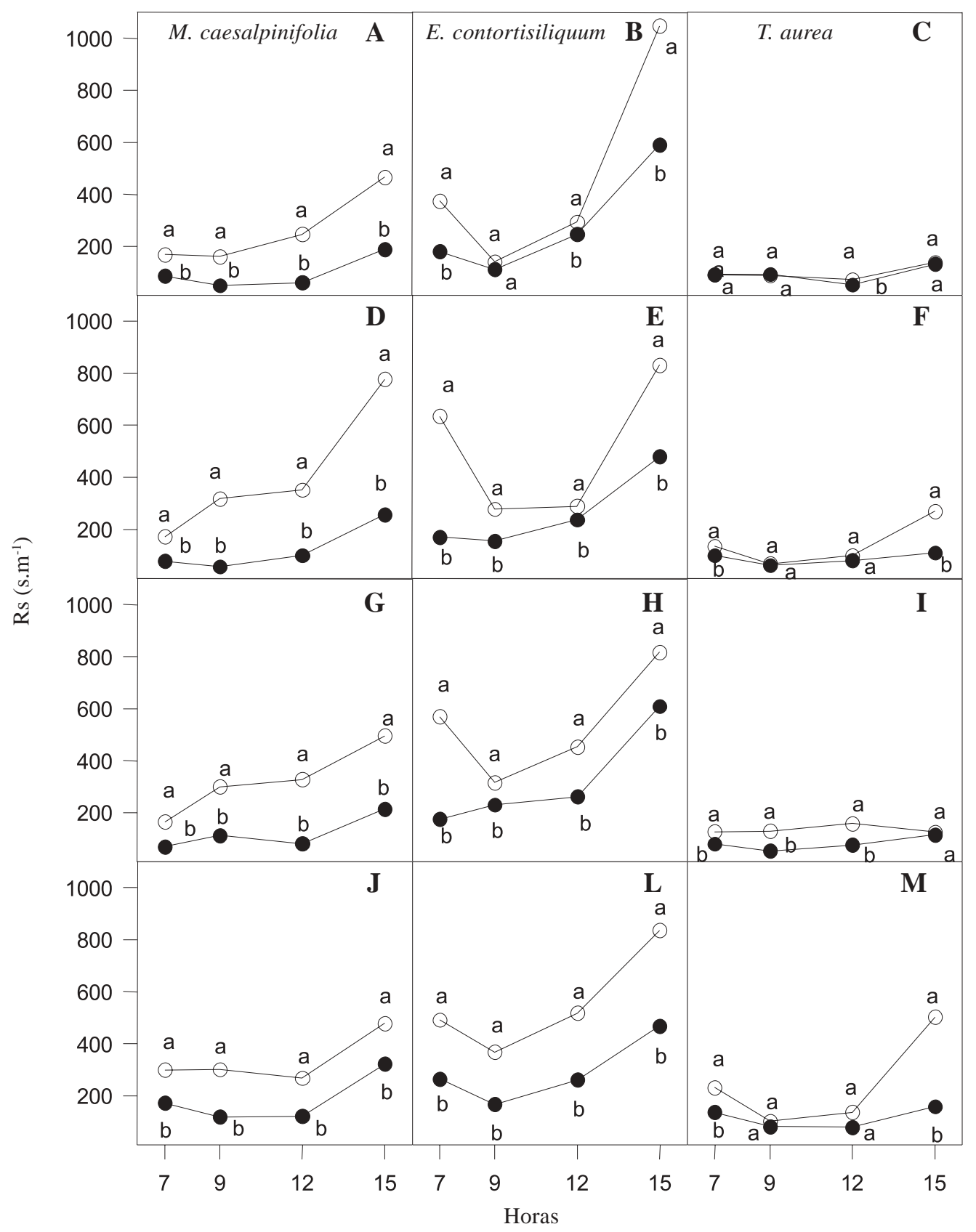

Figura 3. Curso diário da resistência difusiva (Rs) em plantas jovens de Mimosa caesapiniifolia, Enterolobium contortisiliquum e Tabebuia aurea após 5 (A, B, C), 7 (D, E, F), 13 (G, H, I) e 22 (J, L, M) dias de tratamentos hídricos em casa de vegetação. Letras iguais não diferem entre os tratamentos pelo teste de Tukey ao nível de 5\% de probabilidade. 
aos 13 dias, e nas plantas estressadas foi de $1048,75 \mathrm{s.m}^{-1}$. Os valores mais baixos foram registrados entre 7 e 9 horas, sendo de $111,25 \mathrm{s.m}^{-1}$ nas plantas controle e de 141,25 s.m $^{-1}$ nas estressadas, aos 5 dias de estresse.

T. aurea só apresentou diferença significativa na Rs aos 5 dias no horário das 12 horas e aos 7 dias de estresse no horário das $7 \mathrm{e}$ 15 horas, respectivamente. Após 13 dias de tratamento, houve um aumento da Rs nas plantas estressadas nos horários das 7 às 12 horas, não havendo diferença, entretanto, às 15 horas. Já aos 22 dias, a Rs só aumentou nas plantas estressadas às 7 e 15 horas (Fig. 3C, F, I e M). Os valores médios na Rs para essa espécie variaram de $88,0 \mathrm{~s} . \mathrm{m}^{-1}$ a $502,0 \mathrm{~s} . \mathrm{m}^{-1}$ nas plantas estressadas, e de $51,75 \mathrm{~s} . \mathrm{m}^{-1}$ a $158,0 \mathrm{~s} . \mathrm{m}^{-1}$ nas plantas controle (Fig. 3). Não houve correlação significativa entre a Rs e a Tar, UR, PAR e DPV para as plantas estressadas (Tab. 3), porém, foi verificada correlação altamente significativa da Rs com o potencial hídrico foliar (Tab. 6).

Mattos (1992) verificou acentuado aumento na resistência à difusão do vapor de água em folhas de Copaifera langsdorffii, Roupanea umbellata e Xylopia aromatica, em campo, demonstrando que as condições ambientais influenciavam sobre as trocas gasosas nos indivíduos das três espécies. Observou também que, embora tenha havido diferença marcante na resposta à seca, todas as espécies se comportaram de forma a restringir a perda de água através da transpiração.

Em amendoim, Távora \& Melo (1991) observaram acentuado aumento na Rs nas duas superfícies foliares quando as plantas foram submetidas a vários ciclos de estresse por suspensão de rega. Nogueira \& Santos (2000) estudando a mesma espécie verificaram que os efeitos dos diferentes ciclos de estresse hídrico sobre as plantas foi evidenciado através de acréscimos na magnitude dos valores de Rs encontrados, com marcantes diferenças varietais. As autoras ainda observaram três situações distintas, onde uma cultivar manteve os menores valores de Rs durante todo o período de estresse, outra apresentou aumento abrupto da Rs a partir do segundo ciclo, quando o potencial hídrico foliar reduziu-se à -3,9 MPa, e as demais mantiveram-se com pouca variação durante os dois primeiros ciclos, aumentando acentuadamente ao final do último ciclo. Esse último comportamento, bem como o da primeira cultivar, são caracterizados como de resistência à seca.

Nogueira et al.(2000) verificaram aumento da Rs em pitangueiras, com o prolongamento do estresse hídrico, de aproximadamente 70,3\% e $80,2 \%$ para os acessos IPA 4.3 e IPA 2.2 respectivamente. Os autores comentam que o acesso IPA 2.2 se mostrou mais eficiente por reduzir sua transpiração com menor resistência difusiva que o acesso IPA 4.3. Os efeitos da deficiência hídrica no solo na Rs de plantas também foi verificada por Nogueira et al.(2001) em aceroleiras cultivadas em casa de vegetação sob suspensão de irrigação, onde os autores verificaram acréscimos na magnitude dos valores dessa variável com o prolongamento do estresse. Em espécies lenhosas sempre-verdes do campo-sujo e cerradão de Brasília, Naves Barbiero et al. (2000) observaram forte controle estomático da transpiração ao longo do dia no final da estação seca, por não conseguirem absorver água do solo em quantidade suficiente para suprir a alta demanda evaporativa da atmosfera.

No presente trabalho, as plantas de M. caesalpiniifolia e E. contortisiliquum aumentaram e mantiveram os valores de Rs durante todo o período de estresse, denotando uma estratégia para economizar a água existente no solo ao primeiro sinal de redução do seu conteúdo. T. aurea comportou-se de forma diferenciada, mantendo sua resistência semelhante ao das plantas controle durante os primeiros dias de avaliação, elevando a Rs apenas em alguns horários no início da imposição do estresse e aumentando com o 
seu prolongamento, mas com variações. Porém, mesmo com baixos valores de Rs a taxa transpiratória foi reduzida (Fig. 2 e 3). Em espécies da caatinga, Mansur \& Barbosa (2000) encontraram valores que variaram de 129 s.m-1 a 304 s.m-1 em plantas controle e de 947 s.m $^{-1}$ a 1523 s.m $^{-1}$ nas submetidas a estresse hídrico, entre 9 e 10 horas. Esses valores são semelhantes aos encontrados no presente trabalho para as plantas controle, porém bem mais elevados, para esse horário, do que os encontrados para as plantas estressadas. Embora o aumento da Rs em M. caesalpinifolia tenha sido superior a $300 \%$, esses valores ainda são considerados baixos quando comparados com as espécies estudadas por Mansur \& Barbosa (2000), demonstrando que essa diminuição na abertura dos estômatos reduz, mas não impede as trocas gasosas para $M$. caesalpiniifolia, E. cosntortisiliquum e T. aurea. Embora tenha havido diferenças na magnitude dos valores de Rs, as três espécies estudadas utilizaram a redução do poro estomático como estratégia para minimizar a perda de água através da transpiração.

Quanto à temperatura foliar, de forma geral, os maiores valores, independente das espécies e dos tratamentos hídricos, foram observados entre as 9 e 12 horas, horário de maior demanda evaporativa (Fig. 4).

M. caesalpiniifolia não seguiu padrão de comportamento entre as épocas, havendo variações entre horários e tratamentos quanto aos valores. Aos 5 dias, não apresentou diferença significativa entre os tratamentos em nenhum horário. Aos 7 dias, a Tfol das plantas estressadas só esteve acima das controle às 15 horas (Fig. 4, A e D).

Aos 13 dias, verificou-se aumento significativo da Tfol das plantas estressadas com relação às controle, exceto às 9 horas. Aos 22 dias, observou-se aumento da Tfol das plantas estressadas às 7 horas, e às 15 horas as plantas controle apresentaram valores superiores ao das submetidas a estresse (Fig. 4 G e J). A variação da Tfol para esta espécie foi de $27,2{ }^{\circ} \mathrm{C}$ a $31,82{ }^{\circ} \mathrm{C}$ nas plantas controle e de $27,25^{\circ} \mathrm{C}$ a $31,55^{\circ} \mathrm{C}$ naquelas submetidas a estresse.

Para E. contortisiliquum, o estresse hídrico elevou a Tfol nas plantas de forma significativa das 7 às 12 horas, aos 5 e 7 dias de tratamento. Aos 13 dias, a Tfol das plantas estressadas só foi superior a das controle às 7 e 9 horas e aos 22 dias, apenas às 7 e 15 horas (Fig. 4 B, E, H e L). Os valores variaram em média de $28,3{ }^{\circ} \mathrm{C}$ a $31,37{ }^{\circ} \mathrm{C}$ e de $27,9^{\circ} \mathrm{C}$ a $32,1^{\circ} \mathrm{C}$ nas plantas controle e estressadas, respectivamente.

As plantas estressadas de $T$. aurea apresentaram valores de Tfol significativamente maiores do que as plantas controle aos 5 dias, às 7 e 12 horas (Fig. 4C). Aos 7 dias, após diferenciação dos tratamentos hídricos, a Tfol das plantas estressadas esteve acima das controle em todos os horários (Fig. 4F). Aos 13 dias, após o início dos tratamentos hídricos, as plantas estressadas elevaram mais a temperatura do que as controle nos horários mais quentes do dia (9 e $12 \mathrm{~h}$ ), havendo declínio às 15 horas, quando as plantas do tratamento controle apresentaram temperaturas superiores ao das estressadas, provavelmente pela redução na velocidade de absorção de água pelo sistema radicular não atender a demanda evaporativa da atmosfera. Aos 22 dias, só não houve diferença entre os tratamentos para essa espécie às 12 horas, porém os valores de Tfol das plantas controle foram mais elevados do que as estressadas nos horários da manhã. A Tfol para essa espécie variou de $27,05^{\circ} \mathrm{C}$ a $30,8^{\circ} \mathrm{C}$ nas plantas controle e de $26,85^{\circ} \mathrm{C}$ a $31,27{ }^{\circ} \mathrm{C}$ nas submetidas a estresse.

Em soja, Salinas et al. (1996) observaram que aos menores valores de $\mathrm{E}$ a temperatura das folhas foi elevada, processo conhecido como determinante na dissipação de calor. Nogueira et al. (1998a) avaliando as trocas gasosas de três espécies da caatinga, observaram variações de $25,65^{\circ} \mathrm{C}$ a $26,55^{\circ} \mathrm{C}$, em plantas controle e de $25,9^{\circ} \mathrm{C}$ a $27,75^{\circ} \mathrm{C}$, nas estressadas com 20 dias de suspensão de rega. Os autores 


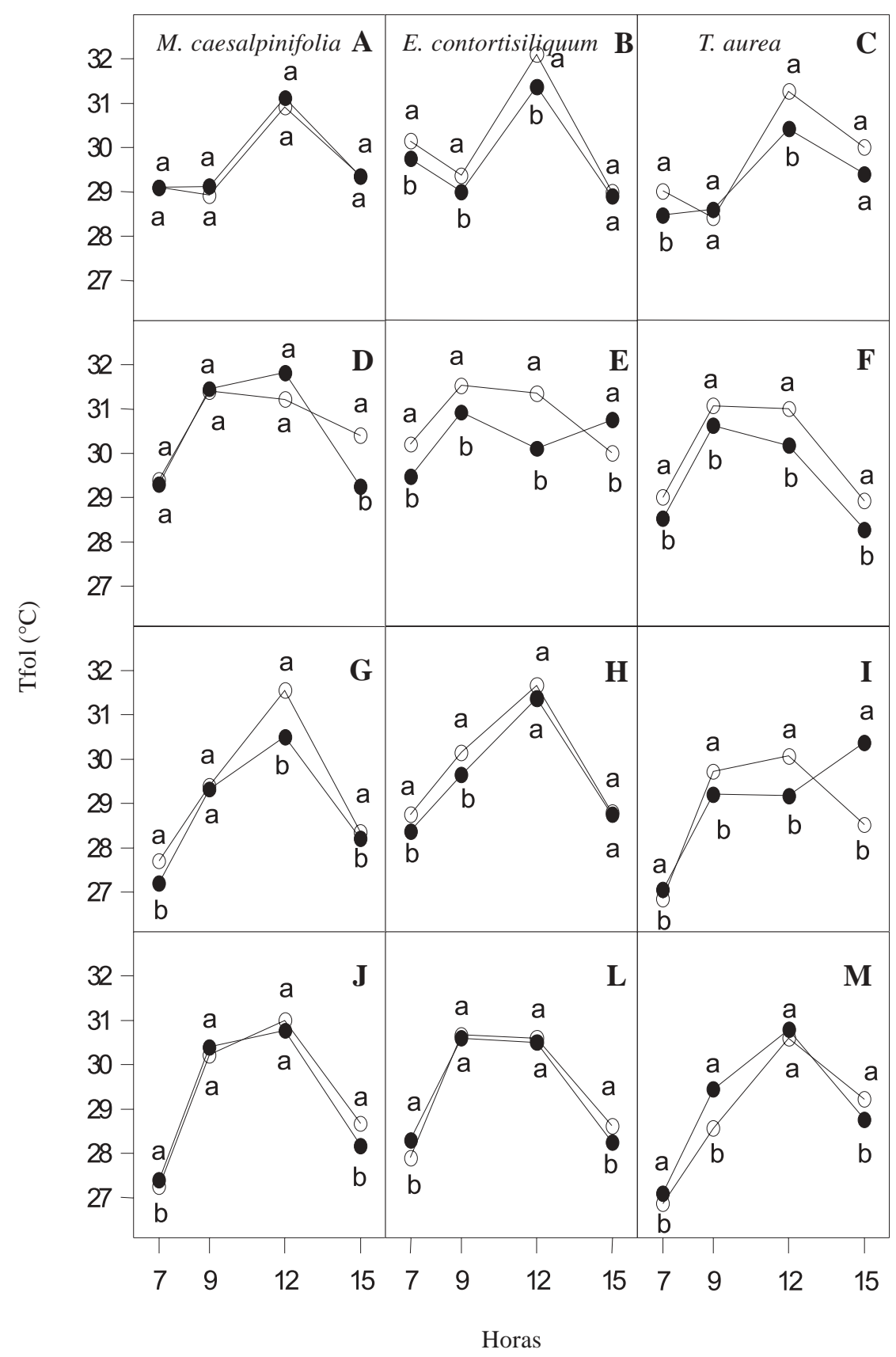

Figura 4. Curso diário da temperatura foliar (Tfol) em plantas jovens de Tabebuia aurea após 5 (A), 7 (B), 13 (C) e 22 (D) dias de tratamentos hídricos em casa de vegetação. Letras iguais não diferem entre os tratamentos pelo teste de Tukey ao nível de 5\% de probabilidade. —— Controle; ——— Estresse. 
verificaram que a Tfol se elevou coincidindo com os menores valores de $\mathrm{E}$ e os maiores de Rs, provavelmente pela dissipação de calor. No presente trabalho a Tfol das plantas de $T$. aurea apresentaram correlação com a E em ambos os tratamentos (Tab. 3). Para M. caesalpiniifolia e E. contortisiliquum apenas as plantas do tratamento controle mostraram essa correlação significativa com E (Tab. 1 e 2).

De forma geral, as condições ambientais contribuíram para o aumento da Tfol em todas as espécies. O DPV influenciou no aumento da Tfol de forma altamente significativa para todas as espécies. Nas plantas submetidas a estresse, o aumento da Tar, bem como do PAR influenciaram no aumento da Tfol, assim como o decréscimo da UR (Tab. 1, 2 e 3). Em virtude da variação apresentada na Tfol, essa variável não se mostrou um bom indicativo de estresse hídrico para a M. caesalpiniifolia, E. contortisiliquum e T. aurea.

Foi observado que, sob estresse hídrico, algumas variáveis climáticas influenciaram na resistência à difusão do vapor de água para a atmosfera. Para M. caesalpiniifolia, o PAR e a UR influenciaram na Rs das plantas sob estresse. Para E. contortisiliquum, apenas a Tar se correlacionou com a Rs nas plantas estressadas. Para T. aurea, apenas a Tar se correlacionou com a Rs das plantas controle, não sendo demonstrada correlação com a UR, PAR e DPV (Tab. 1, 2 e 3).

Alguns pesquisadores têm demonstrado a influência dos fatores climáticos sobre o comportamento estomático de plantas. Gucci et al.(1996) demonstraram a dependência do comportamento estomático de plantas de kiwi sobre o status hídrico do solo e do DPV. Nogueira et al. (1998b) verificaram que não houve correlação entre E e UR em aceroleiras cultivadas na zona semi-árida de Pernambuco, porém encontraram correlação do PAR com a Rs, E, Tfol e $\Psi_{w}$. Em mangabeiras cultivadas no Litoral de Pernambuco, Nogueira et al.(1999) encontraram variação dos efeitos da UR sobre a Tfol, E e Rs em função da posição ocupada pelas folhas na copa (sol e sombra).

Para o potencial da água da folha, a análise de variância revelou efeito significativo tanto para espécie, como para tratamentos hídricos e sua interação (Tab. 4). O estresse hídrico reduziu o potencial da água da folha nas três espécies. Para M. caesalpiniifolia essa redução foi na ordem de $155 \%$, em E. contortisiliquum de $86 \%$ e em T. aurea foi de 40\%. M. caesalpiniifolia apresentou o maior valor de $\Psi \mathrm{w}$, tanto nas plantas controle $(-0,34 \mathrm{MPa})$, como nas plantas estressadas $(-0,87 \mathrm{MPa})$, sendo seguida de E. contortisiliquum com valores médios de $-0,52 \mathrm{MPa}$ e $-0,97$, e $T$. aurea, $-0,71 \mathrm{MPa}$ e $-0,99 \mathrm{MPa}$, para as plantas controle e submetidas a estresse, respectivamente (Tab. 5).

Embora em termos percentuais esses valores sejam significativos, ao se comparar os valores do $\Psi_{\mathrm{w}}$ das espécies estudadas com outras de regiões semi-áridas verifica-se que os valores de $\Psi_{\mathrm{w}}$ da $M$. caesalpiniifolia, E. contortisiliquum e T. aurea, mesmo sob estresse de $50 \%$ da CP são elevados. Nogueira et al. (1998c) avaliaram o potencial hídrico foliar em cinco espécies da caatinga no início da estação seca e encontraram variação nos valores de -0,37 MPa a -3,39 MPa. No intervalo de 12-13 horas os autores registraram valores de $\Psi \mathrm{w}$ de -1,12 MPa para Spondias tuberosa,

Tabela 4. Síntese da análise de variância para o potencial de água da folha $\left(\Psi_{\mathrm{w}}\right)$ em plantas jovens de Mimosa caesalpiniifolia, Enterolobium contortisiliquum e Tabebuia aurea cultivadas em casa de vegetação sob dois regimes hídricos.

\begin{tabular}{lrrrr}
\hline $\begin{array}{l}\text { Fonte de } \\
\text { Variação }\end{array}$ & gl & $\begin{array}{r}\text { Quadrado } \\
\text { médio }\end{array}$ & Valor F & P>F \\
\hline Espécie & 2 & 12,1829 & 38,3915 & 0,000 \\
Tratamento & 1 & 105,8400 & 333,5294 & 0,000 \\
Esp $\times$ Trat & 2 & 2,9787 & 9,3868 & 0,002 \\
Blocos & 3 & 0,3783 & 1,1922 & 0,346 \\
Resíduo & 15 & 0,3173 & & \\
CV $(\%)$ & & 7,67 & & \\
& & & &
\end{tabular}


Tabela 5. Valores médios do potencial da água na folha $\left(\Psi_{\mathrm{w}}\right)$ em plantas jovens de Mimosa caesalpiniifolia, Tabebuia aurea e Enterolobium contortisiliquum, cultivadas em casa de vegetação sob dois regimes hídricos.

\begin{tabular}{lll}
\hline \multirow{2}{*}{ Espécie } & \multicolumn{2}{c}{$\Psi_{\mathrm{w}}(\mathrm{MPa})$} \\
\cline { 2 - 3 } & Controle & Estresse \\
\hline Mimosa caesalpiniifolia & $-0,34 \mathrm{aA}$ & $-0,87 \mathrm{bA}$ \\
Enterolobium contortisiliquum & $-0,52 \mathrm{aB}$ & $-0,97 \mathrm{bAB}$ \\
Tabebuia aurea & $-0,71 \mathrm{aC}$ & $-0,995 \mathrm{bB}$ \\
\hline
\end{tabular}

Médias seguidas de mesma letra minúscula nas linhas e maiúsculas nas colunas, não diferem entre si pelo teste de Tukey a $5 \%$ de probabilidade.

-1,92 MPa para Caesalpinia sincorensis , de 2,12 MPa para Mimosa hostilis, -3,28 para Ziziphus joazeiro e de $-3,39 \mathrm{MPa}$ para Caesalpinia pyramidalis. Nogueira et al. (1998a) também observaram redução no $\Psi \mathrm{w}$ em três espécies da caatinga cultivadas em casa de vegetação. Após 20 dias de suspensão de rega os autores observaram que o potencial diminuiu de -0,88 MPa para -1,98 MPa em Senna martiana, de $-1,18$ para $-3,12$ para Parkinsonia aculeata e de $-0,89$ para $-2,69$ para Senna occidentalis.

Comportamento similar foi verificado em Caesalpinia ferrea, C. pyramidalis, Senna martiana e $S$. spectabilis, com potenciais de $-3,95 \mathrm{MPa},-3,19 \mathrm{MPa},-1,83 \mathrm{MPa}$ e -2,26MPa respectivamente, após 7 dias de suspensão de rega.
Reduções no potencial hídrico foliar em resposta ao estresse hídrico também tem sido observado em plantas do cerrado. Perez \& Moraes (1991) encontraram valores entre -3,5 MPa e -4,0 MPa em espécies arbóreas do cerradão. Em Stryphnodendron adstringens, Rocha \& Moraes registraram valores de até $-2,7 \mathrm{MPa}$, quando a fotossíntese líquida chegou a zero, o mesmo acontecendo com Dalbergia miscolobium, quando o potencial chegou a -2,4 MPa (Sassaki et al. 1997).

Verificou-se correlação inversa entre a Rs e E, da Rs com $\Psi$ w e correlação positiva do $\Psi_{\mathrm{w}}$ com a E e de $\mathrm{E}$ com Tfol nas três espécies estudadas (Tab. 6). Trabalhando com três espécies da caatinga, Nogueira et al. (1998a) também encontraram correlação entre E e Rs e entre E e $\Psi_{w}$ de forma positiva e correlação inversa da Rs com $\Psi$ w e com a Tfol. A mesma correlação inversa foi verificada entre o $\Psi w$ e a Rs em pitangueiras cultivadas sob estresse hídrico em casa de vegetação (Nogueira et al. 2000).

A avaliação do potencial da água na folha, bem como da transpiração e da resistência oferecida pelos estômatos a passagem do vapor de água, demonstraram ser parâmetros relevantes para inferir os efeitos do estresse hídrico sobre M. caesalpiniifolia, E. cfontortisifliquum e T. áurea.

Tabela 6. Coeficiente de correlação simples e probabilidade para a resistência difusiva (Rs), a transpiração (E), o potencial hídrico foliar ( $\Psi$ w), e a temperatura foliar (Tfol), em plantas jovens de Mimosa caesalpiniifolia, Enterolobium contortisiliquum e Tabebuia aurea submetidas a dois regimes hídricos em casa de vegetação.

\begin{tabular}{cccc}
\hline Variáveis & Craibeira & Sabiá & Tamboril \\
\hline Rs X E & $-0,6045^{* *}$ & $-0,7112^{* *}$ & $-0,8413^{* * *}$ \\
Rs X Yw & $-0,9309^{* *}$ & $-0,9721^{* *}$ & $-0,8423^{* *}$ \\
Rs X Tfol & $-0,1849^{*}$ & $0,0033^{\mathrm{NS}}$ & $-0,2997^{* *}$ \\
E X Yw & $0,9494^{* *}$ & $0,9377^{* *}$ & $0,8957^{* *}$ \\
E X Tfol & $0,5612^{* *}$ & $0,3416^{* *}$ & $0,4435^{* *}$ \\
Yw X Tfol & $0,3609^{\mathrm{NS}}$ & $-0,7563^{*}$ & $-0,2016^{\mathrm{NS}}$ \\
\hline
\end{tabular}

* Significativo pelo teste $\mathrm{F}(\mathrm{P}<0,05)$; ** Altamente significativo pelo teste $\mathrm{F}(\mathrm{P}<0,05)$; Ns Não significativo pelo teste $\mathrm{F}$ $(\mathrm{P}<0,05)$ 


\section{Referências bibliográficas}

Andrade Lima, D.1989. Plantas das Caatingas. Academia Brasileira de Ciências.

Braga, R. 1976. Plantas do Nordeste, Especialmente do Ceará. $3^{\text {a }}$ ed. ESAM, Mossoró.

Dias-Filho, M. B. 1995. Root and shoot growth in response to soil drying in four Amazonian weedy species. Revista Brasileira de Fisiologia Vegetal 7(1): 53-59.

Diniz, M. C. M. M. 1999. Desenvolvimento e rebrota da cunhã (Clitoria ternatea $\mathrm{L}$.) sob estrsse hídrico, em associação com fungos micorrízicosBradyrhizobium. Dissertação de Mestrado. Universidade Federal Rural de Pernambuco, Recife.

Ferreira, V. M. 1997. Aspectos de crescimento, nutrição e relações hídricas em dois genótipos de milho (Zea mays L.) em função da disponibilidade de água no solo. Dissertação de Mestrado. Universidade Federal de Lavras, Lavras.

Ferri, M. G. 1979. Transpiração nos principais ecossistemas brasileiros e em espécies cultivadas no Brasil, p.25-73. In: M. G. Fisiologia vegetal. São Paulo: E. P. U.,

Gomes, R. P. 1977. Forragens Fartas na Seca. $4^{\mathrm{a}}$ ed. Nobel, São Paulo.

Gucci, R.; Massai, R.; Xiloyannis, C. \& Flore, J. A. 1996. The effect of drought and vapour pressure déficit on gas exchange of young kiwifruit (Actinidia deliciosa var. deliciosa) vines. Annals of Botany 77: 605-613.

Hopkins, W. G. 1995. Introduction to Plant Physiology. John Wiley \& Sons, Inc., New York.

Judd, W. S.; Campbell, C. S.; Kellogg, E. A. \& Stevens, P. F. 1999. Plant Systematics - A Phylogenetic Approach. Sinauer Associates, Massachusetts.

Krieg, D. R. 1993. Stress tolerance mechanisms in above ground organs. p. 65-79. In: Proceedings of the Workshop on Adaptation of Plants to Soil Stress. INTSORMIL, Nebraska.

Larcher, W. 2000. Ecofisiologia Vegetal. RIMA, São Carlos.

Mansur, R. J. C. N. \& BARBOSA, D. C. A. 2000. Comportamento fisiológico em plantas jovens de quatro espécies lenhosas da caatinga submetidas a dois ciclos de estresse hídrico. Phyton 68: 97-106.

Mattos, E. A. 1992. Trocas gasosas em folhas de três espécies arbóreas do cerradão da fazenda Canchim, São Carlos-SP. Dissertação de Mestrado. Universidade Estadual de São Carlos, São Carlos.
Mendes, B. V. 1986. Alternativas tecnológicas para a agropecuária do semi-árido. $2^{\mathrm{a}} \mathrm{ed}$. Nobel, São Paulo.

Mendes, B. V. 1989. Sabiá (Mimosa caesalpiniifolia Benth.): Valiosa forrageira arbórea e produtora de madeira das caatingas. ESAM, Coleção Mossoroense, série B, n. 660, Mossoró

Naves-Barbiero, C. C.; Franco, A. C.; Bucci, S. J. \& Goldstein, G. 2000. Fluxo de seiva e condutância estomática de duas espécies lenhosas sempre-verdes no campo sujo e cerradão. Revista Brasileira de Fisiologia Vegetal 12(2):119-134.

Nogueira, R. J. M. C.; Barbosa, D. C. A. \& Moraes, J. A. P. V. 1998a. Trocas gasosas e relações hídricas em plantas jovens envasadas de três espécies da caatinga, submetidas a deficiência de água. Phyton 62(1/2): 37-46.

Nogueira, R. J. M. C.; Burity, H. A. \& Moraes, J. A. P. V. 1998b. Transpiração e potencial hídrico foliar em aceroleiras (Malpighia emarginata DC) cultivadas na zona semi-árida de Pernambuco. Revista Científica Rural 3(2): 75-81.

Nogueira, R. J. M. C.; Melo Filho, P. A. \& Araújo, E. L. 1999. Expressões ecofisiológicas de germoplasma de Harconia speciosa Gomes cultivada no litoral de Pernambuco. Ciência Rural 29(4): 731-732.

Nogueira, R. J. M. C.; Melo Filho, P.A. \& Santos, R. C. 1998c. Curso diário do potencial hídrico foliar em cinco espécies lenhosas da caatinga. Revista ecossistema 23: 73-77.

Nogueira, R. J. M. C.; Moraes, J. A. P. V.; Burity, H. A. \& Bezerra Neto, E. 2001. Alterações na resistência à difusão de vapor das folhas e relações hídricas em aceroleiras submetidas a déficit de água. Revista Brasileira de Fisiologia Vegetal 13(1): 75-87.

Nogueira, R. J. M. C.; Santos, R. C.; Bezerra Neto, E. \& Santos, V. F. 1998d. Comportamento fisiológico de duas cultivares de amendoim submetidas a diferentes regimes hídricos. Pesquisa Agropecuária Brasileira 33(12): 1963-1969.

Nogueira, R. J. M. C.; Silva Junior, J. F.; Bezerra, E. F.; Lederman, I. E.; Burity, H. A. \& Santos, V. F. Comportamiento estomático y tensión de água em el xilema de dos genótipos de pianga (Eugenia uniflora L.) cultivados bajo estrés hídrico. 2000. Investigacion Agrária: Production y Proteccion Vegetal 15(3): 49-61.

Perez, S. C. J. G. A. \& Moraes, J. A. P. V. 1991. Determinação de potencial hídrico foliar, condutância estomática e potencial osmótico em espécies dos estratos arbóreo, arbustivo e herbáceo de um cerradão. Revista Brasileira de Fisiologia Vegetal 3(1): 27-37. 
Prado, C. H. B. A.; Moraes, J. A. P. V. \& Mattos, E. A. 1994. Gas exchange and leaf water stress in potted plants of Copaifera langsdorfii. 1. Responses to water stress. Photosynthetica 30(2): 207-213.

Rizzini, C. T. 1986. Árvores e madeiras úteis do Brasil: Manual de dendrologia brasileira. 2a ed. Ed. Edgard Blücher, São Paulo.

Rocha, A. M. S. R. \& Moraes, J. A. P. V. 1997. Influência do estresse hídrico sobre as trocas gasosas em plantas jovens envasadas de Stryphnodendron adstringens (Mart.) Coville. Revista Brasileira de Fisiologia Vegetal 9(1): 43-48.

Salinas, A. R.; Zelener, N.; Craviotto, R. M. \& Bisaro,V. 1996. Respuestas fisiológicas que caracterizan el comportamiento de diferentes cultivares de soja a la deficiencia hídrica en el suelo. Pesquisa Agropecuaria Brasileira 31(5): 331-338.

Santos, R.F. \& Carlesso, R. 1998. Déficit hídrico e os processos morfológicos e fisiológicos das plantas. Revista Brasileira de Engenharia Agrícola e Ambiental 2(3): 287-294.

Sassaki, R. M.; Machado, E. C.; Lagôa, A. M. M. A. \& Felippe, G. M. 1997. Effect of water deficiency on photosynthesis of Dalbergia miscolobium Benth., a cerrado tree species. Revista Brasileira de Fisiologia Vegetal 9(2): 83-87.

Souza, C. C.; Oliveira, F. A.; Silva, I. F. \& Amorim Neto, M. S. 2000. Avaliação de métodos de determinação de água disponível e manejo da irrigação em terra roxa sob cultivo de algodoeiro herbáceo. Revista Brasileira de Engenharia Agrícola e Ambiental 4(3): 338-342.
Scholander, P. F.; Hammel, H. T.; Hemingsen, E. A. \& Bradstreet, E. D. 1964. Hydrostatic pressure and osmotic potencials in leaves of mangroves and some other plants. Proceedings of National Academy Science 51: 119-125.

Távora, F. J. A. F. \& Melo, F. J. 1991. Respostas de cultivares de amendoim a ciclos de deficiência hídrica: crescimento vegetativo, reprodutivo e relações hídricas. Ciência Agronômica 22(1/2): 47-60.

Thomas, D. S.; Eamus, D. \& Shanahan, S. 2000. Influence of season, drought and xylem ABA on stomatal responses to leaf-to-air vapour pressure difference of trees of the Australian wet-dry tropics. Australian Journal of Botany 48: 143-151.

Tigre, C. B. 1964. Guia para o reflorestamento do Polígono das Secas. Ministério da Viação e Obras Públicas. Departamento Nacional de Obras Contra as Secas, Fortaleza.

Vianello, R. L. \& Alves, A. R. 1991. Meteorologia básica e aplicações. Imprensa Universitária da UFV, Viçosa.

Younis, M. E; El-Shahaby, O. A; Hasaneen, M. N. A. \& Gaber, A. M. 1993. Plant growth, metabolism and adaptation in relation to stress conditions: XVII. Influence of different water treatments on stomatal apparatus, pigments and photosynthetic capacity in Vicia faba. Journal of Arid Enviroments 25: 221-232. 